\title{
openheart Heart rate never lies: interventional cardiologist and Braude's quote revised
}

\author{
Stéphane Cook, Jean-Christophe Stauffer, Jean-Jacques Goy, Denis Graf, \\ Serban Puricel, Aurélien Frobert, Olivier Muller, Mario Togni, Diego Arroyo
}

To cite: Cook S, Stauffer J-C, Goy J-J, et al. Heart rate never lies: interventional cardiologist and Braude's quote revised. Open Heart 2016:3:e000373.

doi:10.1136/openhrt-2015000373

Received 4 December 2015 Revised 10 December 2015 Accepted 14 December 2015

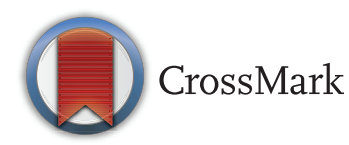

Department of Cardiology, University \& Hospital, Fribourg, Switzerland

Correspondence to Dr Diego Arroyo; da.arroyo@gmail.com

\section{ABSTRACT}

Background: Interventional cardiologists may be immune to stress, allowing them to perform complex percutaneous interventions under pressure.

Objectives: To assess heart rate (HR) variations as a surrogate marker of stress of interventional cardiologists during percutaneous cardiac procedures and in every-day life.

Design: This is a single-centre observational study including a total of six male interventional cardiologists performing coronary interventions and pacemaker implantations. Participants were asked to record their HR with the Apple Watch Device during procedures, every-day life and control activities such as outpatient consultations, sport, marital conflicts and sexual intercourse.

Results: Average daily HR was $88 \pm 17 \mathrm{bpm}$. During work days, HR increased significantly during procedures $(90 \pm 17 \mathrm{bpm})$ compared with days outside the cathlab $(87 \pm 17 \mathrm{bpm}, \mathrm{p}=0.02)$. The average HR was higher during a regular week working $(88 \pm 16 \mathrm{bpm})$ compared with weekends off $(84 \pm 18 \mathrm{bpm}, p=0.002)$. Complex cardiac procedures were associated with higher HR up to $122 \mathrm{bpm}$. Peak HR were higher during physical exertion. Of note, participants complained of hypersexuality and mania after night shifts.

Conclusions: Work and especially percutaneous cardiac procedures increase HR independently of physical exertion suggesting that interventional cardiologists experience mental stress and emotions.

Always behave like a duck keep calm and unruffled on the surface, but paddle like the devil underneath.-Jacob M Braude

\section{INTRODUCTION}

To ensure a successful percutaneous cardiac procedure, the interventional cardiologists need to master a complex set of variables. Multitasking is not natural; it takes countless hours of training and huge dedication. The operator needs to assess vital signs, haemodynamics, handle fluoroscopy equipment, analyse angiographic images, coordinate the team and ensure correct drug administration while running a treatment strategy. The stress endured is considerable, especially when performing interventions on unstable patients,

\section{KEY QUESTIONS}

What is already known about this subject?

- An increase in heart rate is a marker of physical as well as psychological stress, and it may have an impact on survival.

What does this study add?

- Interventional cardiologists experience a significant increase in heart rate during work and especially during percutaneous cardiac procedures. Sleep deprivation and night shifts are associated with mania episodes in certain subjects.

How might this impact on clinical practice?

- Mental stress may have an impact on the survival of interventional cardiologists. The association between mental stress and risk-taking behavior could have an influence on procedural outcomes.

when facing technical difficulties and, of course, in cases of iatrogenic complications. Yet the majority of interventional cardiologists radiate Olympian calm. There are two opposing theories that help explain this peculiarity.

First, the selection process to become an interventional cardiologist is very rigorous and only the gifted get through. Most doctors wish to spend their professional life on call, they crave to get up at 3:00 in the morning and have the privilege to work in a dangerous environment in close contact with blood and radiation. The interventional cardiologist is a superhero; he is cool as a cucumber in both professional and private instances.

But some have different views on the matter and a non-negligible proportion of the medical community sees interventional cardiologists as anosognosic and sometimes ignorant plumbers that unclog arteries. In both theories, the interventional cardiologist appears to lack the emotional substrate for mental stress during cardiac procedures.

The aim of this study was to assess the heart rate (HR) as a stress marker during cardiac procedures in six interventional cardiologists. 


\section{METHODS}

\section{Participants}

Six interventional cardiologists, five performing percutaneous coronary intervention (PCI) and one device implantation, were studied for 2 months.

\section{HR during percutaneous procedures}

The recordings were made on the wrist with the Apple Watch (Apple computer, Cupertino, Caliornia) according to manufacturer instructions. The operators wore the lead garments; the 'work-out' app was selected and activated allowing several recordings per minute. The operator then undertook routine hand disinfection, sterile scrub and gloves covered the body, the arms, the hands and the device. At the end of the procedure, the 'work-out' was stopped and saved, and the app disabled. All operators were asked to keep a logbook (stolen from the hospital reserve for Holter recordings).

\section{HR during control activities}

HR was measured every 30 min according to the Apple protocol. In addition, participants were asked to measure their HR via the 'work-out' app during control activities. These included outpatient visits, everyday life, physical exertion (squash, cycling, running, rock climbing), mental stress (such as giving lectures), or any combination of physical and mental stress (mostly sex or arguing with either official better halves or lovers). The control activities were noted in the aforementioned logbook.

\section{Data extraction}

Following Apple protocol, data were extracted in XML format. At the end of the study period, each participant was asked to open the 'Health' app on his personal smartphone where data were selected and exported. Given the difficulty to transform raw data into statistical variables, a second strategy was developed. Each participant was asked to download the free QS Access App (Quantified Self Lab, USA), select the 'Heart Rate' variable and 'create a table'. A .csv file was then created and opened in Numbers (Apple computer, USA) and Excel (Microsoft corporation, USA).

\section{Statistics}

Continuous variables are reported as means and SDs, or as medians with $25-75 \%$ IQRs according to their distribution. Normality was assessed by visual inspection of histograms and the computation of QQ-plots. Continuous variables were analysed using the Student $t$ test or the Wilcoxon rank-sum test according to their distribution. All statistical analyses were performed using dedicated software (Stata V.13, StataCorp LP, College Station, Texas, USA) at a two-tailed significance level of $\alpha=0.05$.
RESULTS

\section{Baseline characteristics}

Baseline participant characteristics are given in table 1 . All had a considerable experience in interventional cardiology. The operators were arbitrarily classified according to their ages into 'old' if $\geq 60$ years old or 'young' if $\leq 50$ years old. The volume of cardiac interventions is similar to European standards. None of the participants were under any sort of medication and all denied substance abuse (figure 1).

\section{Heart rate}

Average HR was $88 \pm 17 \mathrm{bpm}$. HR was slightly but significantly $(p=0.005)$ higher among the young group (91 $\pm 21 \mathrm{bpm})$ compared with the older group $(87 \pm 15 \mathrm{bpm})$.

\section{Impact of being on call on mean HR}

The average daily HR was highest during cathlab activity $(90 \pm 17 \mathrm{bpm})$ compared with days outside the cathlab $(87 \pm 17 \mathrm{bpm}, \mathrm{p}=0.02)$. There was a mean increase of 3 (1-13) bpm due to cathlab activities compared with normal days. Similarly, the average HR was higher during a regular week working $(88 \pm 16 \mathrm{bpm})$ compared with weekends off $(84 \pm 18 \mathrm{bpm}, \mathrm{p}=0.002)$. There was a average HR gradient: the highest being on-call during the working week $(91 \pm 16 \mathrm{bpm})$ then during normal working days $(88 \pm 16 \mathrm{bpm})$, on-call during the weekend $(86 \pm 18 \mathrm{bpm})$ and finally, during normal weekends (83 \pm 17 bpm; $<<0.0001$ ).

\section{Maximum HR during activities}

Figure 2 shows examples of maximum HR recordings. The highest measurements were recorded during physical activities such as running ( $\left.\mathrm{HR}_{\max } 211 \mathrm{bpm}\right)$, cycling $\left(\mathrm{HR}_{\max } 146 \mathrm{bpm}\right)$ or playing squash $\left(\mathrm{HR}_{\max } 131 \mathrm{bpm}\right)$. Nevertheless, some extreme values were also found during cardiac procedures. Cardiac procedures for 'structural' heart disease, such as intracardiac shunt occlusion $\left(\mathrm{HR}_{\max } 122 \mathrm{bpm}\right)$, transcatheter aortic valve implantation $\left(\mathrm{HR}_{\max } 120 \mathrm{bpm}\right)$ or mitral balloon valvuloplasty $\left(\mathrm{HR}_{\max } 114 \mathrm{bpm}\right)$ were associated with high values. Strikingly and systematically, we noted an increased HR (HR $\left.\max _{\max } 111 \mathrm{bpm}\right)$ a few hours after emergent PCI during the night in a particular participant and during anticipated 'recovery' period. A thorough investigation concluded that these HR peaks were due to sexual intercourse. Similar $\mathrm{HR}_{\max }$ patterns were noted in other participants, but none agreed to spill the beans and reveal the true nature of these very short but intense periods.

\section{DISCUSSION}

The current study on the HR of interventional cardiologists has the following findings: $1 \%$ work increases HR significantly; $2^{\circ} / \mathrm{HR}$ increases especially during procedures in the cathlab; $3^{\circ} / \mathrm{HR}$ increases during cardiac procedures are mostly due to mental stress; $4^{\circ} /$ and are 
Table 1 Baseline characteristics

\begin{tabular}{|c|c|c|c|c|}
\hline & $\begin{array}{l}\text { All } \\
(n=6)\end{array}$ & $\begin{array}{l}\text { Old } \\
(n=2)\end{array}$ & $\begin{array}{l}\text { Young } \\
(n=4)\end{array}$ & p Value \\
\hline Age, year & $51 \pm 8$ & $60 \pm 1$ & $46 \pm 3$ & 0.001 \\
\hline Number of years doing interventional cardiology & $19 \pm 10$ & $31 \pm 1$ & $13 \pm 3$ & 0.0008 \\
\hline Gender & Male & Male & Male & - \\
\hline Sex & Most & NA & Yes & - \\
\hline Number of coronary angiography so far & $8260 \pm 5673$ & $12500 \pm 4950$ & $7430 \pm 1250$ & 0.38 \\
\hline Number of $\mathrm{PCl}$ so far & $4320 \pm 2893$ & $6750 \pm 1838$ & $3730 \pm 642$ & 0.24 \\
\hline Number of coronary angiography during study period & 371 & $63 \pm 22$ & $82 \pm 6$ & 0.41 \\
\hline Number of $\mathrm{PCl}$ during study period & 189 & $31 \pm 7$ & $42 \pm 16$ & 0.36 \\
\hline Number of PM implantation so far & $926 \pm 1068$ & $650 \pm 495$ & $1202 \pm 1694$ & 0.72 \\
\hline Number of pace implanted during study period & 32 & - & $18 \pm 21$ & - \\
\hline Number of ICD, CRT and CRT-D so far & 987 & - & 987 & - \\
\hline Number of ICD implanted during study period & 12 & - & 12 & - \\
\hline Number of children & $4 \pm 1$ & $4 \pm 2$ & $4 \pm 1$ & 1 \\
\hline
\end{tabular}

Values are mean \pm SD.

CRT, cardiac resynchronisation therapy; CRT-D, cardiac resynchronisation therapy and defibrillator; ICD, implantable cardioverter defibrillator; $\mathrm{NA}$, not available; $\mathrm{PCl}$, percutaneous coronary intervention; $\mathrm{PM}$, pacemaker.

of similar magnitude to physical activities such as running, playing squash and often greater than coitus; and $5 \%$ emergent night-time primary PCIs seem to be associated with increased 'rebound' sexual activity in the early morning in some individuals.

\section{Work increases HR}

Our results suggest that working increases mean daytime HR by $10 \%$. Since an increased HR impacts survival, ${ }^{1}$ we can postulate that working decreases survival which is in line with other observations of increased coronary heart disease in individuals working long hours. ${ }^{2}$ Yet common wisdom, such as transmitted by Confucius, stipulates that if you "chose a job you love, (...) you will never have to work a day in your life". Both views are reconciled in the lyrics of French songwriter Henri Salvador who once said in his 1965 hit "Le travail, c'est la santé. Rien faire, c'est la conserver" in other words "work is health, lazing will preserve it".

\section{Cardiac procedures induce significant mental stress among cardiologists}

The device showed minimal physical activity during cardiac procedures, so any increase in HR must have been triggered by mental stress. It appears that the HR increase is inversely proportional to age: operators $<45$ years had a fivefold to eightfold greater HR increase compared with those $>60$ years. One possible hypothesis is therefore that mental stress decreases with age and/or experience.
Figure 1 Examples of recordings in an Interventional Cardiologist's Activities in Daily Life (ICADLs). (A) Cardiac interventions: the footprint of the device (arrow) is visible in the sterile shirt (a sterile glove covering it). (B-D) The device is worn on the wrist during 'on-call' and other activities (here, giving lectures or rock climbing).
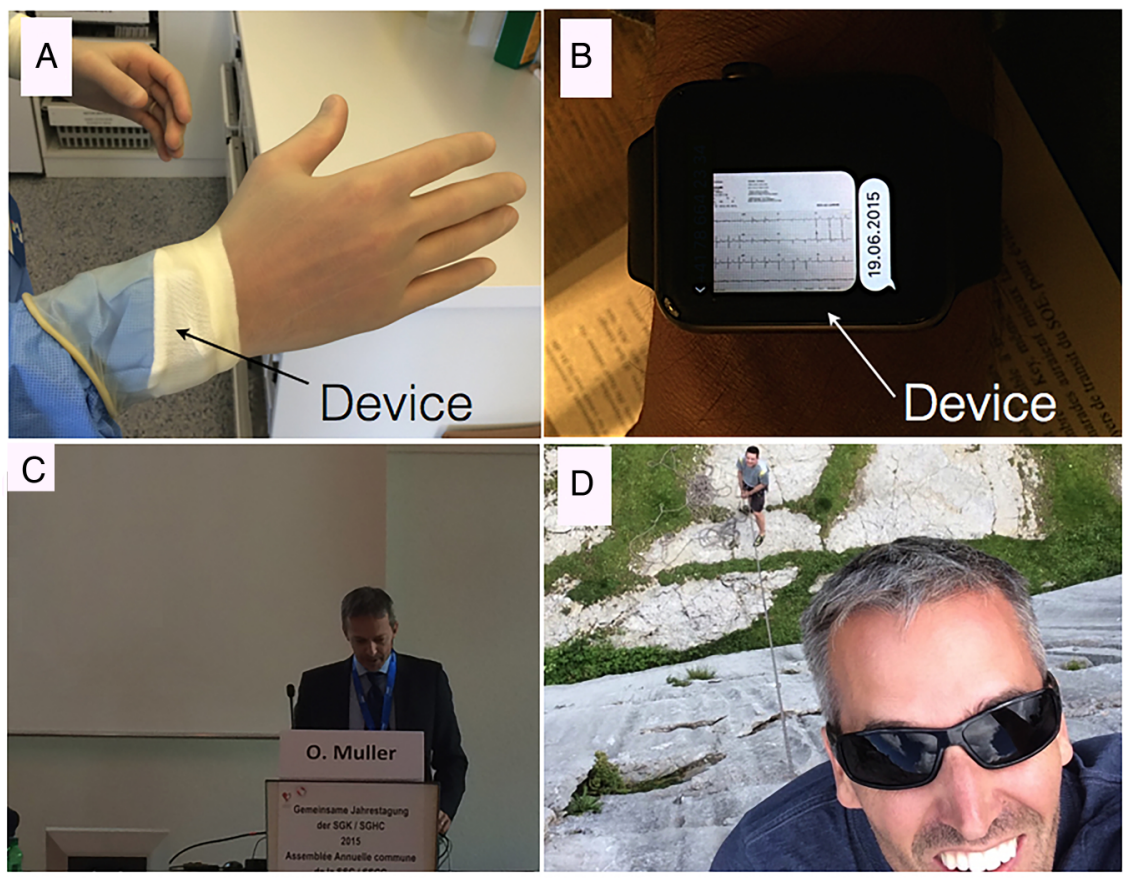


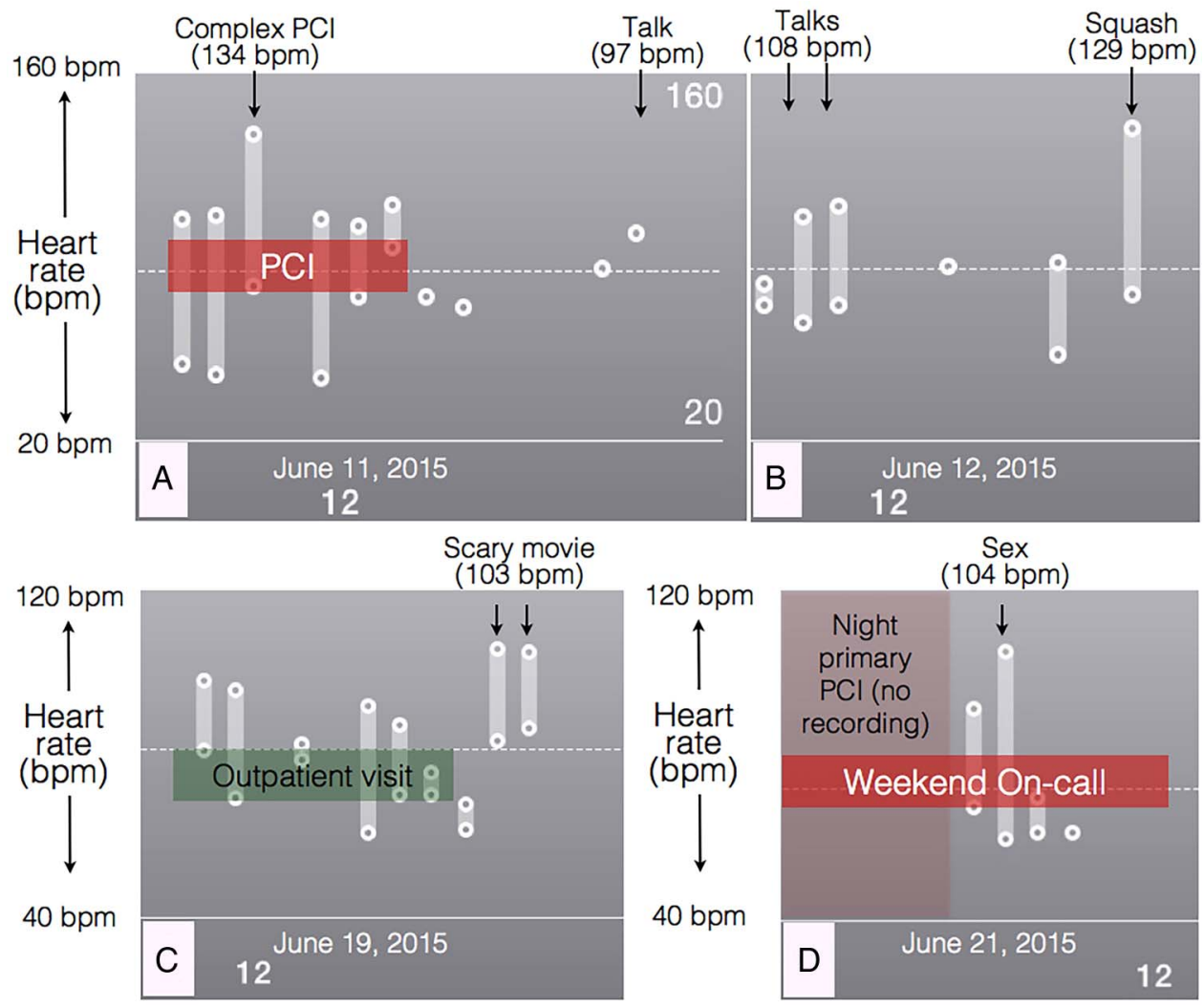

Figure 2 Examples of raw HR recordings on 11 June (A), 12 June (B), 19 June (C) and 21 June (D) in a particular participant. $\mathrm{HR}$ is for heart rate $(\mathrm{PCl}$, percutaneous coronary intervention).

Mental stress can lead to atherosclerotic plaque instability by several mechanisms: it increases sympathetic tone, blood pressure, HR, vascular shear stress, blood viscosity, fibrinogen and von Willebrand factor levels, platelet activity and inflammatory mediators. What better way for the operator to understand and empathise with his patient? HR as a surrogate marker of mental stress not only may influence the operator's health but it may also correlate with procedure success. This thesis is supported by the analysis of London traders by Coates and Herbert ${ }^{3}$ in which stress hormones are incriminated for excessive financial risk taking. Extrapolating the data to cardiac interventions, the HR of the interventional cardiologist could be correlated to inappropriate risks influencing procedure outcomes. This opens a new frame in the world of monitoring where not only patients but also healthcare workers should have their HR measured. Careful attention to $\mathrm{HR}$ variability may predict procedural outcomes.

\section{Night shifts associated with hypersexuality}

One operator had a marked increase in sexual activity after sleep deprivation during night shifts. Other operators also described abnormal elevated mood states and symptoms compatible with idiopathic mania. In addition to hypersexuality, these operators showed signs of hyperactivity, stereotyping and/or irritability. These observations are in line with the sleep deprivation preclinical mania model developed by Gessa et al in rats, ${ }^{4}$ demonstrating a pathophysiological pathway between opioid and dopamine interaction. This model however fails to explain the unusually high birth rate found in our study (2.5× higher than the Swiss average).

\section{Limitations}

The present prospective study has the following strengths: a substantial rate of recordings with few dropouts, its financial independence, and the unique comparison of interventional cardiologist $\mathrm{HR}$ during different activities in everyday life. There are, however, several important limitations. First, given the reduced battery life, no recordings were performed overnight when the devices needed to be loaded. Second, no real periprocedural complications were recorded during the study period. Further studies are needed to assess the operator HR in iatrogeny. Third, the fact that it was performed in a single centre with selected operators with uniform procedural strategies makes generalisations to other centres and other interventional specialties limited. Four, the gender bias precludes any comparison to women performing percutaneous cardiac interventions. Finally, this study did not investigate profession specificity (here interventional cardiologist) on HR. It remains to be proven whether or not other medical (surgeons, obstetricians, anaesthetists, 
etc) or non-medical (traders, pilots, military, etc) professions are at risk.

\section{CONCLUSIONS}

Contrary to common belief, and much like rats and ducks, interventional cardiologists seem to experience stress and appear to have an affective life. This is reassuring. Interventional cardiologists just try to keep calm and carry on.

Acknowledgements The authors are indebted to Dr Andreas Baumbach from the University of Bristol, for his crucial contribution and for drawing our attention to the work of John Coates on London City traders.

Contributors SC conceived and designed the research, handled funding, participated in data acquisition and drafted the manuscript; J-CS participated in data acquisition, and made critical revision of the manuscript and anecdotal quotes such as: "...during the last TAVI procedure, when the fluoroscopy was blocked (inability to see the balloon inflate), my heart rate rose to $120 / \mathrm{min}$, one of the highest rates ever for me, comparable only with when I stare at my wife's overpriced necklace!"; J-JG participated in data acquisition, harassed his son to extract smartphone data, and made critical revision of the manuscript; DG participated in data acquisition and spent a lot of time running; SP analysed the data; AF secured device acquisition abroad and brought them back on Swiss soil despite International Embargo; OM travelled $60 \mathrm{~km}$ to purchase the device, participated in the study and data acquisition and then left on vacation; MT participated in data acquisition and criticised the device throughout the study period; DA participated in data analysis and drafted the manuscript.

Funding This study was funded by the Fonds Scientifique Cardiovasculaire, Fribourg, Switzerland.

Competing interests None declared.

Provenance and peer review Not commissioned; internally peer reviewed.

Data sharing statement No additional data are available.

Open Access This is an Open Access article distributed in accordance with the Creative Commons Attribution Non Commercial (CC BY-NC 4.0) license, which permits others to distribute, remix, adapt, build upon this work noncommercially, and license their derivative works on different terms, provided the original work is properly cited and the use is non-commercial. See: http:// creativecommons.org/licenses/by-nc/4.0/

\section{REFERENCES}

1. Cook S, Togni M, Schaub MC, et al. High heart rate: a cardiovascular risk factor? Eur Heart J 2006;27:2387-93.

2. Virtanen M, Heikkila K, Jokela M, et al. Long working hours and coronary heart disease: a systematic review and meta-analysis. $\mathrm{Am}$ J Epidemiol 2012;176:586-96.

3. Coates JM, Herbert J. Endogenous steroids and financial risk taking on a London trading floor. Proc Natl Acad Sci USA 2008;105:6167-72.

4. Gessa GL, Pani L, Fadda $P$, et al. Sleep deprivation in the rat: an animal model of mania. Eur Neuropsychopharmacol 1995;5 (Suppl):89-93. 